\title{
Artigo/Article
}

\section{Influência da infecção pregressa pelo vírus da hepatite B na fibrose hepática em portadores de hepatite $C$ crônica: avaliação retrospectiva de uma série de casos}

\author{
Influence of previous hepatitis B virus infection on liver fibrosis in patients with chronic \\ hepatitis $\mathrm{C}$ : a retrospective case series evaluation
}

\author{
Gaspar Lisboa Neto ${ }^{1}$, Fatima Mitiko Tengan ${ }^{1}$, Norma de Paula Cavalheiro' ${ }^{1}$ e Antonio Alci Barone ${ }^{1}$
}

\section{RESUMO}

Introdução: A hepatite C é uma das principais causas de doença hepática em todo mundo. Apresenta um curso evolutivo dinâmico e influenciável por diversos co-fatores. Dentre eles, a infecção pregressa pelo vírus B (anti-HBcAg [+] e HBsAg [-]) tem se associado a pior prognóstico histológico e terapêutico. Este trabalho teve como objetivo analisar a associação entre a infecção pregressa pelo vírus $\mathrm{B}$ e fibrose hepática em portadores de hepatite $\mathrm{C}$ crônica, de maneira independente. Métodos: Foram revistos retrospectivamente prontuários médicos de pacientes infectados cronicamente pelo vírus $\mathrm{C}$, atendidos consecutivamente durante um ano no ambulatório de Doenças Infecciosas e Parasitárias - HC FMUSP, quanto aos dados epidemiológicos, clínicos, laboratoriais e histológicos. A análise de independência do impacto da infecção pregressa pelo vírus B foi realizada através de modelo estatístico de regressão logística multivariado, considerando a detecção do anti- $\mathrm{HBcAg}$ como variável de exposição, sendo o desfecho a alteração estrutural histopatológica graus 3 e 4 (septos com formação de nódulos e cirrose). Resultados: 145 indivíduos foram avaliados pelo estudo, $47.2 \%$ com anti-HBcAg (+). O fator de risco mais comumente relatado foi transfusão de sangue e hemoderivados (35,9\%). Embora necrose em saca-bocado tenha sido encontrada com maior frequência no grupo de infecção pregressa, a sorologia anti-HBcAg $(+)$ não se associou à fibrose hepática avançada. Conclusões: A infecção pregressa pelo vírus B não parece acentuar a lesão estrutural desencadeada pela hepatite $C$ crônica, após controle estatístico para outros co-fatores sabidamente capazes de influenciar a história natural desta infecção.

Palavras-chaves: Hepatite C crônica/fisiopatologia. Anticorpos anti-hepatite B. Hepatite B oculta.

\begin{abstract}
Introduction: Hepatitis $\mathrm{C}$ is a major cause of liver disease worldwide. Its evolutionary course is dynamics and may be influenced by several cofactors. Among them, previous hepatitis B virus infection (anti-HBcAg [+] and HBsAg [-]) has been associated with worse histological and therapeutic prognosis. This study had the objective of independently assessing the relationship between previous hepatitis B infection and liver fibrosis in patients with chronic hepatitis C. Methods: The medical records of patients chronically infected with the hepatitis $C$ virus who had been seen consecutively during a one-year period at the infectious and parasitic diseases outpatient clinic of HC FMUSP were retrospectively reviewed in relation to epidemiological, clinical and histological data. Analysis on the independence of the previous hepatitis B infection was performed using the statistical model of multivariate logistic regression. Detection of anti$\mathrm{HBcAg}$ was taken to be the independent variable. The outcome was taken to be grade 3 and 4 histopathological abnormality (septa with nodule formation and cirrhosis). Results: 145 subjects were evaluated in this study. $47.2 \%$ of them were anti-HBcAg $(+)$. The main risk factor for infection was blood and blood derivative transfusion (35.9\%). Findings of anti- $\mathrm{HBcAg}(+)$ were not related to advanced liver fibrosis, although piecemeal necrosis has been found frequently in patients with this serological marker. Conclusions: Previous hepatitis B infection does not seem to increase the structural liver damage triggered by chronic hepatitis $\mathrm{C}$ virus infection, after statistical control for other co-factors capable to impact the natural history of this infection.
\end{abstract}

Key-words: Chronic hepatitis C/physiopathology. Hepatitis B antibodies. Occult hepatitis B.

1. Laboratório de Investigação Médica em Hepatites Virais, Departamento de Doenças Infecciosas e Parasitárias, Hospital das Clínicas, Faculdade de Medicina, Universidade de São Paulo, São Paulo, SP.

Endereço para correspondência: Dr. Gaspar Lisboa Neto. LIM 47/Dept ${ }^{\circ}$ DIP/HC/FMUSP. Av. Dr. Enéas de Carvalho Aguiar 500/1 ${ }^{\circ}$ andar/sala 12, 05403-000 São Paulo, SP.

Tel: $55113085-1601$

e-mail: glneto@usp.br

Recebido para publicação em 26/12/2009

Aceito em 25/05/2010

\section{INTRODUÇÃO}

A hepatite C crônica (VHC) é considerada um grave problema de saúde pública, sendo uma das principais causas de doença hepática em todo mundo. Estima-se que cerca de 160 milhões de pessoas estão cronicamente infectadas pelo vírus $\mathrm{C}$ (3\% da população mundial $)^{1}$. É notável que a grande maioria destes indivíduos encontra-se sobre o risco de desenvolvimento de complicações hepáticas progressivas, como cirrose e hepatocarcinoma ${ }^{2}$.

O curso da doença é dinâmico e altamente influenciado por uma gama de fatores exógenos e endógenos ao hospedeiro ${ }^{3}$. Dentre estes, a avaliação da co-infecção com o vírus da hepatite B (VHB) torna-se relevante, uma vez que se trata de fenômeno comum, visto que ambos os vírus compartilham vias similares de transmissão ${ }^{4}$.

De fato, esta população apresenta marcadores de infecção pregressa pelo vírus B em maior frequência do que na população geral ${ }^{4}$. Estima-se que a prevalência de anti-HBcAg na ausência do antígeno de superfície (HBsAg) em indivíduos com hepatite C crônica seja da ordem de 20 a $30 \%{ }^{5,6}$. No Brasil, um estudo realizado em Campinas, Estado de São Paulo, envolvendo 790 pré-doadores de sangue nos quais os exames de triagem revelaram anti-VHC $(+)$, demonstrou que $30 \%$ dos mesmos apresentavam marcadores de infecção B pregressa ${ }^{7}$.

A hipótese de que os hepatócitos, uma vez infectados, são capazes de transmitir o genoma do vírus $\mathrm{B}$, sob a forma de cccDNA, sugere que a persistência epissomalépossível mesmo emindivíduos com hepatite $\mathrm{B}$ pregressa e resolvida ${ }^{8-11}$. Ainda, a possibilidade da reativação clínica de doença hepática em indivíduos imunocomprometidos e a identificação VHB-DNA através de técnicas de biologia molecular altamente sensíveis no soro, PBMC (células linfomononucleares de sangue periférico) e hepatócitos fortalecem a hipótese de infecção latente e de baixo grau replicativo nesta situação ${ }^{12,13}$. Esta condição tem sido denominada hepatite B oculta ${ }^{14}$. 
O impacto clínico desta infecção silenciosa em pacientes portadores de hepatite $\mathrm{C}$ crônica permanece indefinido, embora a mesma tenha sido correlacionada a um maior dano hepatocelular, aumento de incidência de neoplasia hepática e a menor resposta virológica sustentada no tratamento do vírus $\mathrm{C}^{15,16}$.

Este estudo teve como objetivo avaliar a influência da infecção pregressa pelo vírus B no desenvolvimento de fibrose hepática avançada em pacientes cronicamente infectados pelo vírus da hepatite C, de forma independente aos principais co-fatores sabidamente capazes de interferir na história natural desta infecção.

\section{MÉTODOS}

Foram revistos retrospectivamente prontuários médicos de pacientes diagnosticados com hepatite $\mathrm{C}$ crônica, atendidos de maneira consecutiva em nossa instituição (Ambulatório de hepatites virais - Divisão de doenças infecciosas e parasitárias do Hospital das Clínicas da FMUSP) durante o período de um ano (01/01/2006 a $31 / 12 / 2006)$.

O diagnóstico de hepatite $\mathrm{C}$ crônica foi estabelecido por teste sorológico imunoenzimático de segunda e terceira geração (Abott HCVEIA 2.0 e 3.0), confirmado através da detecção de viremia por metodologia de PCR in-house, baseada na amplicação da região 5’NC viral (sensibilidade: $50 \mathrm{UI} / \mathrm{mL}$ ). A infecção pregressa pelo vírus $\mathrm{B}$ foi definida por critérios sorológicos, sendo considerado o seguinte padrão: anti- $\mathrm{HBcAg}(+)$ e $\mathrm{HBsAg}(-)$, independentemente da detecção do anti-HBsAg (técnica MEIA por kits comerciais Abbot Laboratories Diagnostics Division, North Chicago, IL, USA). Desta maneira, para fins de análise, os indivíduos portadores de hepatite $\mathrm{C}$ crônica avaliados foram estratificados em dois grupos: com infecção pregressa (anti-HBcAg [+] \pm anti-HBsAg) e sem infecção pregressa (anti-HBcAg [-]).

Os critérios de inclusão adotados pelo estudo foram: idade acima de 18 anos, infecção crônica pelo vírus C, perfil sorológico disponível para vírus $\mathrm{B}$ e presença da análise anatomopatológica hepática, sendo a amostra considerada satisfatória pelo Departamento de Anatomia Patológica do HC-FMUSP (contendo ao menos 10 espaços-porta). Foram excluídos pacientes infectados pelo HIV, com infecção crônica ou ativa pelo vírus $\mathrm{B}$ (HBsAg detectável), com hepatopatias de origem autoimune ou metabólica e com histórico de tratamento prévio para hepatite C. Os registros de indivíduos com exames laboratoriais realizados além do período de 74 semanas, antes ou após a data da realização da biópsia hepática, também não foram considerados.

Os seguintes dados epidemiológicos e clínicos foram registrados: gênero, idade à época da realização da biópsia hepática, provável fonte de infecção (transfusão sanguínea ou de hemoderivados anterior a 1992, procedimento cirúrgico ou invasivo, uso de droga injetável ou inalatória, tatuagem, piercing, comportamento sexual de risco, exposição ocupacional, contato intrafamiliar não-sexual, etilismo e tabagismo acentuados (equivalente a ingestão de $>40 \mathrm{~g}$ de etanol/dia para homens e $20 \mathrm{~g} /$ dia para mulheres; $>20$ anos/maço, respectivamente) e presença de comorbidades. As variáveis laboratoriais constituíam: exame de genotipagem do vírus C por técnica INNO-LIPA (Versant, Siemmens, USA), dosagem de transaminases (AST e ALT), enzimas canaliculares (fosfatase alcalina e gamaglutamiltranspeptidase), bilirrubinas, albumina, índice internacional normatizado (INR), contagem de leucócitos e de plaquetas.
A biópsia hepática foi realizada através de punção percutânea guiada por ultrassonografia, em regime de hospital-dia. A amostra foi fixada, submetida à microtomia e corada com hematoxilinaeosina e tricômio de Masson para a análise histopatológica. A equipe de patologistas foi informada sobre o diagnóstico de hepatite $\mathrm{C}$ crônica, através de formulário próprio. $\mathrm{O}$ critério classificatório adotado por este estudo foi o do Consenso Nacional sobre a Classificação das Hepatites Crônicas das Sociedades Brasileiras de Patologia/Hepatologia ${ }^{17}$.

\section{Análise estatística}

Os dados nominais foram descritos através de suas frequências absolutas e relativas. Os dados contínuos tiveram suas médias aritméticas calculadas como medidas de tendência central, juntamente com os respectivos desvios-padrão como medidas de dispersão. Algumas variáveis contínuas foram categorizadas em dois ou mais grupos, permitindo o reconhecimento das frequências de cada um, a citar: idade à data da biópsia estratificada em decênios na etapa descritiva, idade $>$ ou < que 40 anos na análise bivariada, AST $>$ ou $<$ que 40UI/L, ALT $>$ ou $<$ que 40UI/L, bilirrubinas totais $>$ ou $<$ que $1.10 \mathrm{mg} / \mathrm{dL}$, albumina $>$ ou $<$ que $3.5 \mathrm{~g} / \mathrm{dL}$, INR $>$ ou $<$ que 1.20 , leucometria $>$ ou $<$ que $5.000 / \mathrm{mm}^{3} \mathrm{e}$ contagem de plaquetas $>$ ou $<$ que $150.000 / \mathrm{mm}^{3}$. O teste do quiquadrado de Pearson foi utilizado para a análise de significância estatística na comparação de proporções no momento em que os indivíduos foram separados em 2 grupos de acordo com a presença ou ausência de infecção pregressa pelo vírus $\mathrm{B}$.

Para a avaliação de possíveis preditores de fibrose hepática acentuada (variável dependente), correspondente a alteração estrutural graus 3 e 4 no exame anatomopatológico, realizou-se análise estatística por regressão logística não condicional univariada, num primeiro momento. Dentre as variáveis explanatórias supracitadas, avaliou-se também a influência da atividade necroinflamatória hepática, sendo aqui representada pela atividade periportal maior ou igual a 2 (presença de necrose em saca-bocado). Aquelas que apresentaram $\mathrm{p}<0,20$ foram incluídas na análise multivariada de independência, realizada através do modelo de regressão logística sequencial. A variável de estudo anti- $\mathrm{HBcAg}(+)$, ou seja, aquela representativa do grupo com infecção pregressa pelo VHB, foi considerada independentemente do seu grau de significância na primeira análise. Inicialmente, todas as variáveis foram incluídas neste modelo. Aquelas que quando testadas não apresentaram significância estatística, baseada no teste de razão de verossimilhança, foram removidas sequencialmente. As razões de chances (Odds ratio) bem como seus respectivos intervalos de confiança a $95 \%$ foram calculados como estimativa dos riscos relativos.

O modelo final foi aquele que apresentou menor discrepância em relação às frequências observadas e estimadas, através do teste de Hosmer e Lemeshow ${ }^{18}$. Sua capacidade de determinação para a fibrose acentuada foi obtida através do cálculo do $\mathrm{R}^{2}$ de Nagelkerke ${ }^{19}$. A avaliação da significância de cada coeficiente da função logística obtida foi realizada através do teste de Wald ${ }^{19}$. Determinou-se a acurácia discriminatória do modelo através do cálculo da área sobre a curva ROC (Receiver Operating Characteristic) ${ }^{20}$.

Os valores calculados para o $\mathrm{p}$ foram bicaudados, sendo considerado o valor menor que 0,05 estatisticamente significativo. A análise dos dados foi realizada com o software SPSS versão 15.0 (SPSS Inc, 2006). 


\section{Ética}

O seguinte projeto foi enviado ao Comitê de Ética em Pesquisa do Departamento de Doenças Infecciosas e Parasitárias e do Hospital das Clínicas da Faculdade de Medicina de São Paulo, sendo aprovado em ambos (Protocolo de Pesquisa número 0738/07 - CAPPesq HC-FMUSP).

\section{RESULTADOS}

Cento e quarenta e cinco registros médicos foram avaliados pelo estudo. As características epidemiológicas e clínicas destes pacientes estão descritas na Tabela $\mathbf{1}$.

Houve uma distribuição proporcional dos casos em relação ao gênero e à idade na data de realização de biópsia hepática, estratificada em decênios. $O$ fator de risco mais frequentemente relatado para infecção pelo vírus $\mathrm{C}$ foi transfusão de sangue e hemoderivados (35,9\%). Trinta e cinco (24\%) indivíduos relataram exposição a duas ou mais situações de risco e aproximadamente $30 \%$ dos indivíduos desconheciam alguma situação predisponente de infecção em sua história clínica. A hipertensão arterial sistêmica foi a comorbidade mais comum, descrita em 17\% dos casos. A análise genotípica do vírus $C$ estava disponível em 125 registros. O genótipo 1 foi o mais $(74,4 \%)$ prevalente, seguido pelos genótipos 3 e 2 ( $16 \%$ e $8,8 \%$, respectivamente).

No total, os achados histopatológicos da análise do fragmento hepático revelaram um predomínio de hepatite leve/moderada em relação ao estadiamento da fibrose. Cento e dezessete (80,7\%) amostras apresentaram pontuação de $0-2$ na alteração estrutural. Todavia, a atividade periportal $\geq 2$ esteve presente em $67,6 \%$ dos indivíduos.

Do grupo total de pacientes portadores de hepatite $\mathrm{C}$ crônica avaliados, sessenta e dois $(42,7 \%)$ pacientes apresentavam o marcador de infecção pregressa pelo vírus B. A análise bivariada quanto ao gênero, idade e exames laboratoriais, agrupados de acordo com o status sorológico do anti-HBcAg ([+] ou [-]), não revelou diferenças estatisticamente significativas entre os dois grupos, embora tenha ocorrido tendência a um maior número de indivíduos acima de 40 anos no grupo de infecção pregressa $(p=0,050)$. Entretanto, necrose em saca-bocado foi encontrada em maior frequência no grupo de infecção pregressa quando comparados com o grupo anti-HBcAg (-) (Tabela 2).
As variáveis selecionadas para a análise uni e multivariada de possíveis preditores de fibrose hepática avançada estão relatadas na Tabela 3. As variáveis gênero masculino, idade acima de 40 anos, etilismo acentuado, bilirrubina total, INR acima de 1.20 e atividade periportal $\geq 2$ perderam significância na análise multivariada. A presença do anti-HBcAg manteve-se sem significância estatística. Desta forma, foram considerados preditores independentes de fibrose hepática avançada neste estudo: tabagismo acentuado, aumento de ALT, aumento de $\gamma$-gt e leucócitos abaixo de 5.000/mm3 (Tabela 3 ).

TABELA 1 - Características clínicas e epidemiológicas de pacientes portadores de hepatite C crônica - DMIP - HC FMUSP.

\begin{tabular}{lcc}
\hline Característica & $\begin{array}{c}\text { Frequência absoluta } \\
\text { no(total) }\end{array}$ & $\begin{array}{c}\text { Frequência } \\
\text { relativa (\%) }\end{array}$ \\
\hline Gênero masculino & $76(145)$ & 52,4 \\
Idade à data da biópsia & & \\
$18-30$ & $38(145)$ & 26,2 \\
$31-40$ & $28(145)$ & 19,3 \\
$41-50$ & $43(145)$ & 26,7 \\
$>$ 50 & $36(145)$ & 24,8 \\
Fonte de infecção & & \\
transfusão de sangue/derivados & $52(145)$ & 35,9 \\
cirurgia/procedimento invasivo & $41(145)$ & 28,3 \\
comportamento sexual de risco & $11(145)$ & 7,6 \\
uso de droga inalatória & $10(145)$ & 6,9 \\
tatuagem/piercing & $10(145)$ & 6,9 \\
uso de droga injetável & $9(145)$ & 6,2 \\
contato intrafamiliar não-sexual & $8(145)$ & 5,5 \\
exposição ocupacional & $4(145)$ & 2,8 \\
desconhecido & $41(145)$ & 28,3 \\
Comorbidades & & \\
hipertensão & $25(145)$ & 17,2 \\
diabetes mellitus & $9(145)$ & 6,2 \\
hipotireoidismo & $6(145)$ & 4,1 \\
porfiria cutânea tarda & $4(145)$ & 2,8 \\
infecção pelo HTLV 1/2 & $3(145)$ & 2.1 \\
\hline & & \\
\hline
\end{tabular}

TABELA 2 - Análise comparativa entre pacientes portadores de hepatite C crônica de acordo com o status sorológico anti-HBcAg - DMIP - HC FMUSP.

\begin{tabular}{|c|c|c|c|}
\hline & $\begin{array}{c}\text { Anti-HBcAg }(+) \\
\pm \text { anti-HBsAg }\left(n^{\circ}=62\right)\end{array}$ & $\begin{array}{c}\text { Anti-HBcAg (-) } \\
\left(\mathbf{n}^{\mathrm{o}}=83\right)\end{array}$ & \\
\hline Característica & $\mathrm{n}=(\%)$ & n⿳o(\%) & $\mathbf{p}$ \\
\hline Idade $>40$ anos* & $43(69,4)$ & $44(53,0)$ & 0,050 \\
\hline \multicolumn{4}{|l|}{ Exames laboratoriais } \\
\hline $\mathrm{ALT}<40 \mathrm{UI} / \mathrm{L}$ & $23(37,1)$ & $24(28,9)$ & 0,298 \\
\hline $\mathrm{BT}<1.10 \mathrm{mg} / \mathrm{dL}$ & $51(82,3)$ & $72(86,7)$ & 0,456 \\
\hline $\mathrm{ALB}>3.5 \mathrm{~g} / \mathrm{dL}$ & $60(96,8)$ & $83(100,0)$ & 0,181 \\
\hline INR $<1.20$ & $56(90,3)$ & $70(84,3)$ & 0,291 \\
\hline LEUCO $>5.000^{* *}$ & $52(83,9)$ & $67(80,7)$ & 0,625 \\
\hline PLT $>150.000^{* *}$ & $56(90,3)$ & $79(95,2)$ & 0,253 \\
\hline $\mathrm{AE}$ graus 3 e 4 & $11(18,8)$ & $17(20,5)$ & 0,679 \\
\hline $\mathrm{APP} \geq 2$ & $50(80,6)$ & $48(57,8)$ & 0,004 \\
\hline
\end{tabular}

*idade na data da biópsia hepática, ${ }^{* *} / \mathrm{mm}^{3}$,

AST: aspartatoaminotransferase, ALT: alaninoaminotransferase, BT: bilirrubina total, ALB: albumina, LEUC: leucócitos, PLT: plaquetas, AE: alteração estrutural hepática, APP: atividade periportal. 
TABELA 3 - Fatores associados à fibrose hepática avançada em pacientes portadores de hepatite C crônica de acordo com modelos de regressão logística uni e multivariados - DMIP - HC FMUSP.

\begin{tabular}{|c|c|c|c|c|c|c|}
\hline \multirow[b]{2}{*}{ Características } & \multicolumn{3}{|c|}{ Análise univariada } & \multicolumn{3}{|c|}{ Análise multivariada } \\
\hline & $n^{\circ}(\%)$ & OR (IC 95\%) & $\mathbf{p}$ & OR & IC $95 \%$ & $\mathbf{p}$ \\
\hline Gênero masculino & $76(52,4)$ & $2,22(0,93-5,31)$ & 0,073 & - & - & - \\
\hline Idade $>40$ anos & $83(57,2)$ & $2,66(1,05-6,74)$ & 0,034 & - & - & - \\
\hline Tabagismo acentuado & $24(16,6)$ & $2,52(0,69-0,95)$ & 0,063 & 3,50 & $1,01-12,17$ & 0,048 \\
\hline Etilismo acentuado & $13(9,0)$ & $2,96(0,88-9,88)$ & 0,077 & - & - & - \\
\hline Anti-HBcAg (+) & $62(42,7)$ & $0,84(0,36-1,94)$ & 0,679 & 0,38 & $0,11-1,24$ & 0,110 \\
\hline $\operatorname{AST}(\mathrm{UI} / \mathrm{L})^{*}$ & $49,7( \pm 39,8)$ & $1,04(1,02-1,06)$ & $<0,001$ & - & - & - \\
\hline $\operatorname{ALT}(\mathrm{UI} / \mathrm{L})^{*}$ & $66,6( \pm 50,1)$ & $1,02(1,01-1,03)$ & $<0,001$ & 1,01 & $1,00-1,03$ & 0,007 \\
\hline$\Gamma$-gt $(\mathrm{g} / \mathrm{dL})^{*}$ & $63,9( \pm 58,1)$ & $1,01(1,00-1,02)$ & $<0,001$ & 1,01 & $1,00-1,02$ & 0,047 \\
\hline $\mathrm{BIL}(\mathrm{mg} / \mathrm{dL})$ & $0,78( \pm 0,3)$ & $3,07(1,02-9,26)$ & 0,046 & - & - & - \\
\hline $\operatorname{ALB}(\mathrm{g} / \mathrm{dL})^{*}$ & $4,36( \pm 0,4)$ & $0,75(0,26-2,15)$ & 0,595 & - & - & - \\
\hline INR $>1.20$ & $17(11,7)$ & $3,57(1,22-10,43)$ & 0,020 & - & - & - \\
\hline LEUC $<5.000 / \mathrm{mm}^{3}$ & $26(17,9)$ & $4,40(1,73-11,17)$ & 0,002 & 6,18 & $1,61-23,60$ & 0,008 \\
\hline $\mathrm{PLT}<150.000 / \mathrm{mm}^{3}$ & $11(7,6)$ & $15,20(3,71-62,22)$ & $<0,001$ & 6,41 & $0,93-44,30$ & 0,059 \\
\hline Atividade periportal $\geq 2$ & $98(67,5)$ & $8,12(1,83-35,90)$ & 0,006 & 4,11 & $0,77-21,75$ & 0,096 \\
\hline
\end{tabular}

*Média \pm Desvio padrão, AST: aspartatoaminotransferase, ALT: alaninoaminotransferase, $\gamma$-gt: gamaglutamiltranspeptidase, BIL: bilirrubina total ALB: albumina, LEUC: leucócitos, PLT: plaquetas, OR: Odds ratio, IC: intervalo de confiança, Nota: teste de Hosmer and Lemeshow: $\chi^{2}=6.654 . p=0.574, R^{2}$ Nagelkerke=0,50; AUROC: $88,5 \%$ (IC95\%: 82,40-94,50).

\section{DISCUSSÃO}

A infecção crônica pelo vírus da hepatite $C$ não é incomum no Brasil ${ }^{7}$. Como a hepatopatia viral tem curso evolutivo extremamente heterogêneo, o esclarecimento das condições que levam à instalação de doença terminal é de fundamental importância. A análise conjunta do fator hipotético, neste caso a infecção pregressa pelo vírus B, com elementos sabidamente capazes de influenciar sua história natural, como o sexo, idade e o uso abusivo de álcool, é necessária para que conclusões equivocadas sejam evitadas.

A amostra em estudo foi composta proporcionalmente por homens e mulheres. Oprincipal fator de risco relatado relacionado àinfecção pelovírus C foi a transfusão de sangue e hemoderivados, uma vez que considerou-se somente os procedimentos realizados antes do início da década de 1990, quando os programas de biossegurança e de triagem sorológica para este vírus ainda eram insatisfatórios. Quanto às comorbidades, as mais frequentemente relatadas forma hipertensão arterial e diabetes mellitus, não se diferenciando do padrão geral da comunidade.

$A$ infecção pregressa pelo vírus $B$, ou seja, a presença do marcador sorológico anti- $\mathrm{HBcAg}$, não se correlacionou à fibrose hepática acentuada em portadores de hepatite $\mathrm{C}$, mesmo com maior frequência de necrose em saca-bocado neste grupo de pacientes. De forma semelhante, Myers e cols ${ }^{21}$ não identificaram diferenças relativas ao anti-HBcAg quanto à lesão estrutural avançada, bem como maior taxa de progressão de fibrose em uma amostra de 132 indivíduos ( 51 com infecção pregressa pelo vírus B). Nosso achado vai contra a teoria da manutenção residual do vírus B neste cenário, sugerindo desta forma a ausência de seus epítopos em hepatócitos, ao menos em quantidades suficientemente capazes de induzir uma maior resposta citotóxica e fibrogênica, ou mesmo pautando a hipótese da supressão não citolítica de sua suposta replicação viral, exercido por células $T$ através da liberação de citoquinas capazes de suprimir a transcrição de seu genoma sem causar dano celular ${ }^{22}$.

Embora não realizada neste estudo, a diferenciação entre o padrão anti-HBcAg isolado e o perfil sorológico completo (anti-HBcAg[+] e
anti-HBsAg[+]) para a avaliação da influência da infecção pregressa pelo VHB na hepatopatia do vírus $\mathrm{C}$ foi proposta por alguns autores, partindo do pressuposto de que a primeira situação estaria associada a um maior dano hepático ${ }^{6,23}$. Contudo, neste ponto a literatura tem demonstrado resultados conflitantes. Enquanto Giannini e cols ${ }^{23}$ descreveram uma maior frequência de marcadores de infecção pregressa pelo vírus $\mathrm{B}$, em especial do padrão anti-HBcAg isolado, em pacientes cirróticos, De Maria e cols ${ }^{6}$, Wedemeyer e cols ${ }^{24}$ e CarvalhoFilho e cols ${ }^{16}$ demonstraram o mesmo desfecho em pacientes anti$\mathrm{HBcAg}$, independente da detecção do anti-HBsAg.

A análise multivariada demonstrou que o tabagismo acentuado, o aumento de ALT, de y-gt e a leucopenia estavam associados à fibrose hepática avançada. Dentre os hábitos de vida, o tabagismo vem ganhando espaço devido seu impacto negativo na doença hepática. Acredita-se que seu efeito deletério seja resultante de sua ação imune secundária ao stress oxidativo e a peroxidação de lipídeos, provocados pela nicotina e tetracloreto de carbono ${ }^{25}$. Ainda, os pacientes portadores de doença hepática crônica, em especial os cirróticos, frequentemente apresentam aumento de enzimas hepáticas e podem evoluir com citopenias em qualquer uma das três séries hematológicas, secundárias ao hiperesplenismo ${ }^{26}$.

Este estudo apresentou algumas limitações, como a própria avaliação retrospectiva de prontuários médicos, além dos possíveis vieses de informação e da indefinição do tempo de co-infecção e sequência de infecção, ou seja, de qual vírus foi adquirido inicialmente (VHC ou VHB). Naquelas situações em que a infecção pregressa pelo VHB ocorreu mesmo antes do vírus $\mathrm{C}$, a história natural da hepatite $\mathrm{C}$ crônica poderia ter sido pouco influenciada pela suposta ação residual do VHB, uma vez que o clareamento do mesmo pelo hospedeiro se daria em uma condição imunológica diferente do ambiente próinflamatório encontrado na hepatopatia $\mathrm{C}$ crônica.

Embora seja metodologicamente desafiador, a detecção intrahepática do genoma do vírus $\mathrm{B}$, em especial de suas formas intermediárias replicativas continua sendo considerada o padrão ouro para o diagnóstico de infecção oculta ${ }^{27}$. Aqui não foi realizada tal pesquisa, devido ao tipo de delineamento adotado, incompatível com a análise proposta. 
Do ponto de vista histológico, a grande maioria dos pacientes avaliados era portadora de doença hepática de leve a moderada intensidade. Desta forma, não é possível afirmar a reprodutibilidade dos resultados caso o grupo em estudo fosse composto primordialmente por indivíduos cirróticos ou em transformação nodular. Além disso, é possível que a amostra avaliada não tenha sido numericamente adequada para a demonstração das diferenças esperadas quanto à presença de fibrose acentuada/cirrose, de acordo com o status anti$\mathrm{HBcAg}$, uma vez que o cálculo da amostra estimada não foi realizado na fase de planejamento.

Em conclusão, a infecção pregressa e resolvida pelo vírus da hepatite $\mathrm{B}$ em pacientes portadores de hepatite $\mathrm{C}$ crônica não esteve associada a um maior dano estrutural hepático em nossa série de casos, sugerindo a ausência de uma suposta atividade imunopatogênica residual e persistente do vírus B capaz de acentuar a atividade fibrogênica promovida pelo vírus $\mathrm{C}$.

\section{AGRADECIMENTOS}

Aos profissionais responsáveis pela assistência aos pacientes do Ambulatório de Hepatites Virais do Departamento de Doenças Infecciosas e Parasitárias/HC/FMUSP.

\section{CONFLITO DE INTERESSE}

Os autores declaram não haver nenhum tipo de conflito de interesse no desenvolvimento do estudo.

\section{REFERÊNCIAS}

1. Guirao AA, Yuste SR, Regueiro BJ. Epidemiología y manifestaciones clínicas de las hepatitis virales. Enferm Infecc Microbiol Clin 2006; 24:264-276.

2. Shiffman ML. Chronic hepatitis C. Semin Liver Dis 2004; 24(suppl 2):1-2.

3. Pawlotsky JM. Pathophysiology of hepatitis $\mathrm{C}$ virus infection and related liver disease. Trends Microbiol 2004; 12:96-102.

4. Dehesa-Violante R, Nuñez-Nateras R. Epidemiology of hepatitis virus B and C. Arch Med Res 2007; 38:606-611.

5. Cacciola I, Pollicino T, Squadrito G, Cerenzia G, Orlando ME, Raimondo G. Occult hepatitis $B$ virus infection in patients with chronic hepatitis $\mathrm{Cliver}$ disease. N Engl J Med 1999; 341:22-26.

6. De-Maria N, Colantoni A, Friedlander L, Leandro G, Idilman R, Harig J, et al. The impact of previous HBV infection on the course of chronic hepatitis C. Am J Gastroenterol 2000; 95:3529-3536.

7. Gonçales-Jr FL. Avaliação clínicoepidemiológica prospectiva de candidatos a doadores e sangue anti-HCV reagentes. Rev Soc Bras Med Trop 1999; 32:315-317.

8. Laras A, Koskinas J, Dimou E, Kostamena A, Hadziyannis SJ. Intrahepatic levels and replicative activity of covalently closed circular hepatitis B virus DNA in chronically infected patients. Hepatology 2006; 44:694-702.

9. Lin ZM, Yatsuhashi H, Daikoku M, Hamada R, Nakao R, Fukuda M, et al. Hepatitis $\mathrm{B}$ virus of genotype $\mathrm{C}$ persistence after recovery from acute hepatitis $B$ virus infection in Japan. Hepatol Res 2003; 25:244-253.

10. Michalak TI, Pasquinelli C, Guilhot S, Chisari FV. Hepatitis B virus persistence after recovery from acute viral hepatitis. J Clin Invest 1994; 94:907.

11. Zhu Y, Yamamoto T, Cullen J, Saputelli J, Aldrich CE, Miller DS, et al. Kinetics of hepadnavirus loss from the liver during inhibition of viral DNA synthesis. J Virol 2001; 75:311-322.

12. Mason AL, Xu L, Guo L, Kuhns M, Perrillo RP. Molecular basis for persistent hepatitis B virus infection in the liver after clearance of serum hepatitis B surface antigen. Hepatology 1998; 27:1736-1742.
13. Rehermann B, Ferrari C, Pasquinelli C, Chisari FV. The hepatitis B virus persists for decades after patients' recovery from acute viral hepatitis despite active maintenance of a cytotoxic T-lymphocyte response. Nat Med 1996; 2:1104-1108.

14. Raimondo G, Pollicino T, Cacciola I, Squadrito G. Occult hepatitis B virus infection. J Hepatol 2007; 46:160-170.

15. Carreño V, Bartolomé J, Castillo I, Quiroga JA. Occult hepatitis B virus and hepatitis C virus infections. Rev Med Virol 2008; 18:139-157.

16. Carvalho-Filho RJ, De-Lucca-Schiavon L, Narciso-Schiavon JL, Sampaio JP, Lanzoni VP, Gomes Ferraz ML, et al. Clinical and histological impact of previous hepatitis B virus infection in patients with chronic hepatitis C. Liver Int 2009; 29:133-140.

17. Gayotto L. Comitê SBP/SBH. Visão histórica e consenso nacional sobre a classificação das hepatites crônicas. Projeto do Clube de Patologia Hepática da Sociedade Brasileira de Patologia aprovado pela Sociedade Brasileira de Patologia. Gastroenterol Endoscopia Dig 2000; 19:137-141.

18. Hosmer JR, Lemeshow S. Applied logistic regression. New York: John Wiley \& Sons; 1989.

19. Bewick V, Cheek L, Ball J. Statistics review 14: Logistic regression. Crit Care 2005; 9:112-118.

20. Martinez EZ, Louzada-Neto F, Pereira BB. A curva ROC para testes diagnósticos. Cad Saude Coletiva 2003; 11:7-31.

21. Myers RP, Thibault V, Poynard T. The impact of prior hepatitis B virus infection on liver histology and the response to interferon therapy in chronic hepatitis $\mathrm{C}$. J Viral Hepat 2003; 10:103-110.

22. Rehermann B. Immune responses in hepatitis B virus infection. Semin Liver Dis $2003 ; 23: 21-38$.

23. Giannini E, Ceppa P, Botta F, Fasoli A, Romagnoli P, Ansaldi F, et al. Previous hepatitis $B$ virus infection is associated with worse disease stage and occult hepatitis B virus infection has low prevalence and pathogenicity in hepatitis $\mathrm{C}$ virus-positive patients. Liver Int 2003; 23:12-18.

24. Wedemeyer H, Cornberg M, Tegtmeyer B, Frank H, Tillmann HL, Manns MP. Isolated anti-HBV core phenotype in anti-HCV-positive patients is associated with hepatitis C virus replication. Clin Microbiol Infect 2004; 10:70-72.

25. Pessione F, Ramond MJ, Njapoum C, Duchatelle V, Degott C, Erlinger S, et al. Cigarette smoking and hepatic lesions in patients with chronic hepatitis $\mathrm{C}$. Hepatology 2001; 34:121-125.

26. Streiff MB, Mehta S, Thomas DL. Peripheral blood count abnormalities among patients with hepatitis C in the United States. Hepatology 2002; 35:947-952.

27. Sagnelli E, Imparato M, Coppola N, Pisapia R, Sagnelli C, Messina V, et al. Diagnosis and clinical impact of occult hepatitis B infection in patients with biopsy proven chronic hepatitis C: a multicenter study. J Med Virol 2008; 80:1547-1553. 